\title{
Using L-M BP Algorithm Forecase the 305 Days Production of First-Breed Dairy
}

\author{
Xiaoli Wei, Guoqiang Qi*, Weizheng Shen, and Sun Jian \\ Agricultural engineer center, Northeast Agricultural University, \\ Harbin, Heilongjiang Province, China, 150030, \\ Tel.: +0451-55191146; Fax: +86-0451-55191146 \\ liuhaiyang790214@163.com
}

\begin{abstract}
Aiming at the shortage of conventional BP algorithm, a BP neural net works improved by L-M algorithm is put forward. On the basis of the network, a Prediction model for 305 day's milk productions was set up. Traditional methods finish these data must spend at least 305 days, But this model can forecast first-breed dairy's 305 days milk production ahead of 215 days. The validity of the improved BP neural network predictive model was validated through the experiments.
\end{abstract}

Keywords: Dairy Milk production, prediction Neural Networks, BP algorithm$\mathrm{M}$ algorithm.

\section{Introduction}

Milk yield is the main indexes to reflect and measure the cow's performance, and it is generally measured by a standard of 305 days milk yield in the world, But in the breeding work, we must calculate the milk production of 305 days, whether determining the descendants of bulls or assessing the quality of cows, which will take a long time and then extend the select cycle, thus how to use the cow's early productive performance to judge it good or not, and speed up the process of selection response has become the concerned and important topic(Qin zhirui,2005).

In this paper, A prediction model based on L-M BP neural network will be introduced to use in estimating the dairy 305 days milk quantities (Jiaoji Cheng, 1992), significantly, the model not only can forecast 305 days milk quantities production of the first-breed cow ahead of 215 days but also speed up the process of breeding dairy.

\subsection{Improved BP Algorithm}

Traditional BP algorithm uses the steepest gradient descent method to amend the weights, training course from starting point to reach the minimum point gradually along the slop of error function to reach iterate of each time, As for the complex network, error surface is in the multi -dimensional space, will therefore possibly fall

\footnotetext{
* Corresponding author.
} 
into some- local smallest dot in the training process, so that will be unable to restrain, in addition, on the one hand, the stability of traditional BP stable request study rate is very small in the, therefore, the gradient descent law makes the training process very slow, on the other hand, the learning rate of momentum method usually be quicker than the pure gradient descent method, however, it is still slow in the practical application, all of above, two methods are only usually suitable for the increment training (Yin chaotian,2005).

This thesis will bring up the new method Levenberg-Marquardt training method which based on the characters of nonlinear system and considered to improve the traditional BP network, Levenberg-Marquardt training method can decrease sensitivity of the network regarding the erroneous surface local detail, and can limit the network to fall into the local minimum effectively, its basic idea is no longer along the sole antigradient direction to iterate of each time .but it permit error carry on the search along the worsened direction, greatly enhance the convergence rate and the pan-ability of network. This algorithm is much quicker than trainbp and train-bpx function's gradient descent law, but need more memories (Wang yu, 1992).

\section{Prediction Model for First-Breeding Dairy}

\subsection{Sample Deal and Realize}

Using the survey data which come from Heilongjiang province about the first breeding cow primary production and extracting 200 data recordings mainly include cow's maximum daily production, the 90 days and the 305 days production, Take 100 randomly selected records as the learning and training sample, other 100 cows records are used as forecast model examination sample, because the data range of variation is wide and the dimension is different, but the input and output based on neural network should be limited in certain scope, making the biggest input still to fall in the big place of neuron transforms function gradient, this may speed up the training speed of network, and can make the network training to be more effective, so it must be standardized for the network.

\subsection{Network Design}

In order to build up the neural network model, we firstly need to determine the neural network structure of forecast model, using three-layer neural network structure (Wen xin,Zhou lu, 2005):Input, Hidden-layer, Output .After building model, using this model can obtain cow's 305 days milk production on Output. The number of input nodes $(\mathrm{N})$ is related with the input sample pattern. If the cow's highest milk production and 90 days milk production are took as the input pattern, then the number $(n)=2$, taking the cow's 305 days milk production as the output of network, because the samples in this article are few, and only by an implicit strata, many strata nodes must be established, the established strata nodal point number is $6 \mathrm{n}$, therefore,the network architecture of forecasting model is $n \times 6 n \times 1$, namely $2 \times 12 \times 1$. The transfer function of input node uses sigmoid hyperbolic tangent as formula (1)

$$
\mathrm{f}(\mathrm{x})=\operatorname{th}(\mathrm{x})=\frac{\mathrm{e}^{\mathrm{x}}-\mathrm{e}^{-\mathrm{x}}}{\mathrm{e}^{\mathrm{x}}+\mathrm{e}^{-\mathrm{x}}}
$$


The transfer function of output node uses the linear function as formula (2).

$$
f(x)=x
$$

\subsection{Analysis}

To forecast the cow's 305days milk production by improved BP network, under Matlab programming environment, network setting allow to make the max error is 0.01, the largest circulation is 10000 times, After training in 3904 iteration step-bystep, consuming one minute and three seconds, the network is restrain, then we use the traditional bp network to test samples(100 first-breeding cows ), compare with the milk production between predicted production and actual production in 305 days milk yield, we can find the max error of network is about $77 \mathrm{~kg}$, the smallest error is about5 $\mathrm{kg}$.

In order to reflect the superiority of improved BP( L-M BP) or traditional BP algorithm, fig.1 and fig. 2 give the network's convergence situation of improved and traditional BP network in the same experimental data. As shown in fig 2 about the

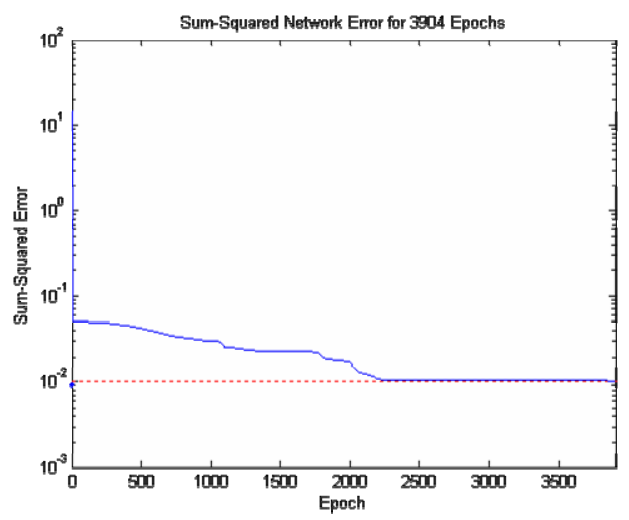

Fig. 1. Improved BP network

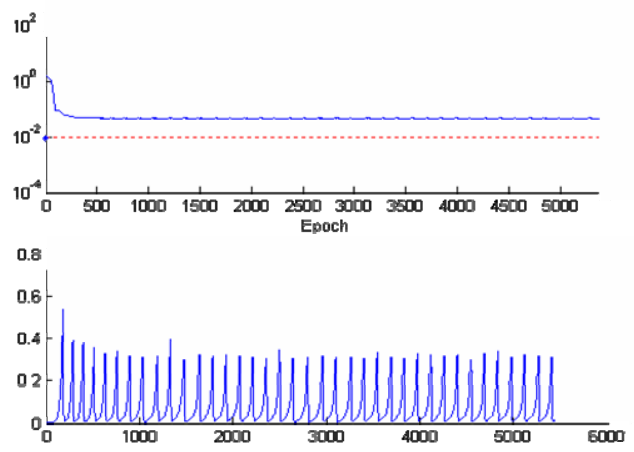

Fig. 2. Traditional BP network 
traditional BP network, the SSE is no longer to renew when training achieves the certain extent, and the slackness of network appeared, causing the network not to go on training, but the improved BP network can overcome this question, it can jump out local minimum by regulating network parameter constantly, thus the network could be trained rapidly(Wei xiaoli,2006).

\section{Experiments}

L-M BP will not only improve the speed but also to improve the operation stability, we use L-M BP for the three months testing in several farms, the following example to illustrate this point, Through the forecast about twenty first-breeding cows' 305 days milk production from Mudanjiang Hailing dairy farm, 8511 cattle farm and XiangFang cattle farm, the result as shown in table1.

Table 1. The result of the forecast model

\begin{tabular}{|c|c|c|c|c|c|c|}
\hline num & CowID & $\begin{array}{l}\text { the maximum } \\
\text { daily } \begin{array}{l}\text { milk } \\
\text { production }\end{array}\end{array}$ & $\begin{array}{l}\text { the } 90 \text { days } \\
\text { production } / \mathrm{kg}\end{array}$ & $\begin{array}{l}\text { the } 305 \text { days } \\
\text { production } / \mathrm{kg}\end{array}$ & $\begin{array}{l}\text { the forecasting } \\
\text { production of } 305 \text { days } \\
/ \mathrm{kg}\end{array}$ & error $/ \mathrm{kg}$ \\
\hline 1 & 491007 & 20.2 & 1541 & 4597 & 4641 & 44 \\
\hline 2 & 20004001 & 28.3 & 2213 & 6977 & 7047 & 70 \\
\hline 3 & 20004002 & 15.2 & 1026 & 3841 & 3852 & 11 \\
\hline 4 & 20004003 & 22.1 & 1713 & 5758 & 5826 & 68 \\
\hline 5 & 20004005 & 32.3 & 2143 & 6780 & 6687 & 7 \\
\hline 6 & 7977 & 20.6 & 1351 & 5020 & 4997 & -23 \\
\hline 7 & 7822 & 28.1 & 2286 & 6836 & 6798 & -38 \\
\hline 8 & 7945 & 28.6 & 1930 & 7045 & 6978 & -67 \\
\hline 9 & 7936 & 22.3 & 1717 & 5755 & 5832 & 77 \\
\hline 10 & 7969 & 28 & 2115 & 6065 & 6070 & 5 \\
\hline
\end{tabular}

\section{Conclusions}

To use the forecast model, we can forecast first-breeding dairy's 305 days milk production when the dairy has just begin to product for three months( 90 days), in other word we will forecast the cow's production ahead of 215 days, by this method, you can choose a good milk production dairy in favor of breeding work.

It will supply us the condition for the advantaged of choosing excellent cows. It is known from the experiment, although this forecast model also has certain error, but it in the allowed scope, it is totally feasible to create a nonlinear system forecast model about cow's 305 days milk production with improved BP network in the production. 


\section{Acknowledgements}

This paper is supported by the animal science center of northeast agricultural university, dairy experimental data, Experimental information and experimental methods are all coming from the topics "dairy science and technology major project" subject No is 2006BAD04A09, thanks of topic groups' helping, significantly, I want to express my thankful for Harbin Intelligent Agricultural Center of Northeast Agricultural University. Xiang-Fang Farm, Animal Medicine Center of Northeast Agricultural University.

\section{References}

Zhirui, Q.: Introduction of dairy cattle breeding guidance, pp. 1-5. Jindun Press, Beijing (2005) Cheng, J.: Neural network introduction, vol. 32, pp. 22-28. Xi'an University of Electronic Science and Technology Publish, Xi' an (1992)

$\mathrm{Yu}, \mathrm{W} .:$ The prediction model and using of the improved BP model. Compute Measurement and Control 13(1), 39 (2005)

Xin, W., Lu, Z.: Matlab neural network using and design. Science Publish, Beijing

Chaotian, Y.: Artificial intelligence and expert system, Beijing

Changhong, D.: Matlab network design and application, vol. 54, pp. 23-28. Science Publish, Beijing (2004)

Xiaoli, W., Yonggen, Z.: Using BP forecast the first-breeding dairy's 305 production. Beijing, China dairy (7), 24-25 (2006) 